\title{
Strongly Coupled Outer Squircle-Inner Circular Coil Architecture for Enhanced Induction Over Large Areas
}

\author{
Veli Tayfun Kilic, Student Member, IEEE, Emre Unal, Erdal Gonendik, Namik Yilmaz, \\ and Hilmi Volkan Demir, Senior Member, IEEE
}

\begin{abstract}
This paper reports a newly designed class of strongly coupled planar coil structures for the purpose of enhanced induction over large areas. These new architectures feature a squircle shape at the outer rim with rounded corners and straight sides evolved into a fully circular shape in the inner side, which proves to be essential to achieve high efficiency in arrays and all-surface inductive heating. As a proof-of-demonstration, a simple inductive heating system composed of a pair of side-by-side placed coils was constructed together with a ferrite layer. Experiments were repeated for $0^{\circ}$ and $180^{\circ}$ phase differences between coil currents. Here, the system efficiency was shown to be increased overall by $37.4 \%$ using outer squircle-inner circular coils instead of conventional circular coils. This comparative study indicates that the proposed coil architecture offers the potential for large-area, fast, and phase-insensitive inductive heating with high efficiency.
\end{abstract}

Index Terms-Coils, energy efficiency, induction heating, inductive power transmission, wireless energy transfer.

\section{INTRODUCTION}

$\mathbf{E}$ LECTRICITY is increasingly more commonly used in industrial and household applications. An important utilization of electricity is heating and cooking systems. Although all of them use electricity as the power source, different types exist, including conventional resistive and inductive heating. Resistive heating is the most common technique, but these systems are neither safe nor efficient. On the other hand, recently inductive heating has been becoming increasingly more popular, especially in East Asian and European countries because of

Manuscript received December 22, 2015; revised March 20, 2016 and May 25, 2016; accepted June 13, 2016. Date of publication July 27, 2016; date of current version November 8, 2016. This work was supported in part by Arcelik A.S. and in part by the Industry Theses Program SAN-TEZ 1463-STZ-2012-2.

V. T. Kilic and E. Gonendik are with the Department of Electrical and Electronics Engineering, Bilkent University, Ankara 06800, Turkey (e-mail: vkilic@ bilkent.edu.tr; erdal@ee.bilkent.edu.tr).

E. Unal is with the Institute of Materials Science and Nanotechnology, Bilkent University, Ankara 06800, Turkey (e-mail: unale @ bilkent.edu.tr).

N. Yilmaz is with Arcelik A.S. Company, Arcelik Tuzla Campus, Istanbul 34950, Turkey (e-mail: namik.yilmaz@ arcelik.com).

H. V. Demir is with the Department of Electrical and Electronics Engineering, the Department of Physics, and the Institute of Materials Science and Nanotechnology, Bilkent University, Ankara 06800, Turkey, and also with the School of Electrical and Electronic Engineering and the School of Physical and Mathematical Sciences, Nanyang Technological University, Singapore 639798 (e-mail: volkan@ bilkent.edu.tr)

Color versions of one or more of the figures in this paper are available online at http://ieeexplore.ieee.org

Digital Object Identifier 10.1109/TIE.2016.2594228 its safety, cleanliness, and quick warming and high-efficiency capabilities [1]. All these advantages of inductive systems result from the fact that electromagnetic energy is directly transferred to the vessel floor and the heat is produced right there.

Despite all of the advantages of induction-based heating, there are still pending problems in induction hobs. One of the problems is switching losses caused by high-frequency inverter module. To decrease such losses, different inverter topologies have been developed [2]-[4]. Another problem observed in induction heaters (and also in other types of cookers) is efficient heating of different shaped and sized vessels. In conventional induction cookers, specific heating regions exist on the surface. When a small-sized vessel (e.g., a coffee pot) is placed, which does not cover the coil beneath the surface, power transfer efficiency from the coil to the vessel substantially degrades. This can be explained with the flux produced from an uncovered area of the coil. In addition, for efficient energy transfer, a vessel should ideally be placed right in the middle of the coil. These situations restrict users in terms of both the usage area and the number of vessels that can be inductively heated simultaneously.

To inductively heat different sized vessels, concentric coils have been previously proposed and widely exploited [5]-[8]. Although these coils enable more efficient heating of vessels in varying sizes than conventional coils, complete flexibility is still not possible. For efficient inductive heating, vessels having similar sizes with the inner and/or outer coils should be used. Also, vessels should be placed again right on top of the coil.

With the aim of enabling complete flexibility in terms of size, location, and number of simultaneously cooking vessels, arrays of small coils are used in induction cookers. Such systems have been studied for various perspectives both theoretically and experimentally [8]-[13]. In these previous reports, arrays of coils with only traditional shapes, including circular, elliptical, square, and rectangular coils, have been investigated to date. Although circular shaped coils can be stacked into a more compact array by hexagonal placement, coil-to-coil interaction and coupling between these coils in the array are not strong enough as a result of the large curvatures on their outer rims. On the other hand, although they provide long interaction sides, square- and rectangle-shaped coils unnecessarily require longer wires than the curved coils to occupy the same area. In addition, because of their sharp corners, square and rectangular coils are not easy to produce and their couplings at the corners can be undesirable. 


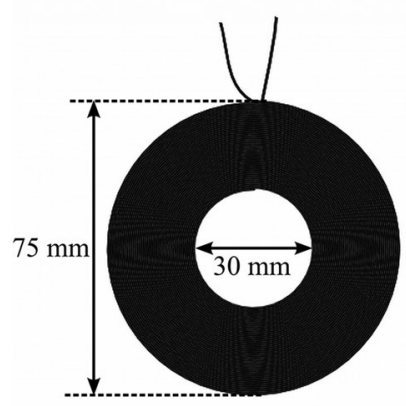

(a)

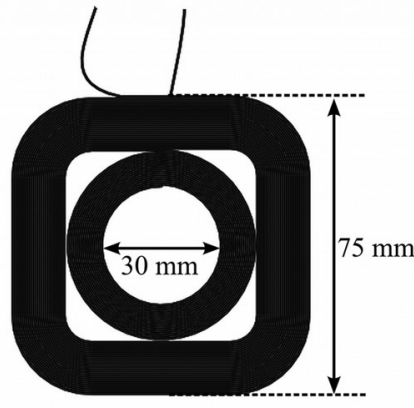

(b)
Fig. 1. Top-view illustration of coil structures: (a) the conventional circular and (b) the proposed outer squircle-inner circular coil architectures.

In this paper, different from the previous studies, we propose and demonstrate a new class of coil architectures that feature squircle windings at the outer rim with fully rounded corners and long straight sides evolving into circular windings at the inner rim. This coil structure uniquely allows for strong coupling in arrays and easy manufacturing at the same time. Also, the proposed outer squircle-inner circular coils are adaptable to phase difference applications because they exhibit high efficiencies with and without phase differences. Here, we present the design and characterization of our proposed outer squircle-inner circular coils and compare their efficiency with the conventional circular coils experimentally. Furthermore, we demonstrate the relative efficiency improvements by applying the signal to the coupled coils with $0^{\circ}$ and $180^{\circ}$ phase differences. The demonstrated outer squircle-inner circular coils prove to be beneficial to address the aforementioned problems of an induction cooker with large areas.

\section{Coll Architecture And Experimental Setup}

Fig. 1 shows schematics of the manufactured coil structures: the conventional circular coil as a reference structure in Fig. 1(a) and the proposed outer squircle-inner circular coil structure in Fig. 1(b) as the new architecture to be studied. A conventional circular coil is selected as a reference for comparison because it is the most commonly used geometry in all-surface inductive heating systems based on its properties stated above. The inner diameter and the outer projection side length of these structures are fixed to 30 and $75 \mathrm{~mm}$, respectively. These lengths are selected to match the minimum vessel dimensions accepted by some manufacturers. In our outer squircle-inner circular coils, the straight parts constitute $35 \mathrm{~mm}$ of the side length, while the total turns of both the manufactured coils are 44 . In the newly designed coil, there exist 18 circular turns inside and 26 squircle turns outside, adding up to a total of 44 turns, and these turns are connected to each other such that continuous current circulates in the same direction both inside and outside. In both coils, each turn is composed of 13 copper wire strands with $0.250 \mathrm{~mm}$ diameter. The radius of the used wire strands $(0.125 \mathrm{~mm})$ is within the skin depth at operational frequencies of induction heating systems. Also, the selected number of strands allows for the maximum current limit of the electric fuses in

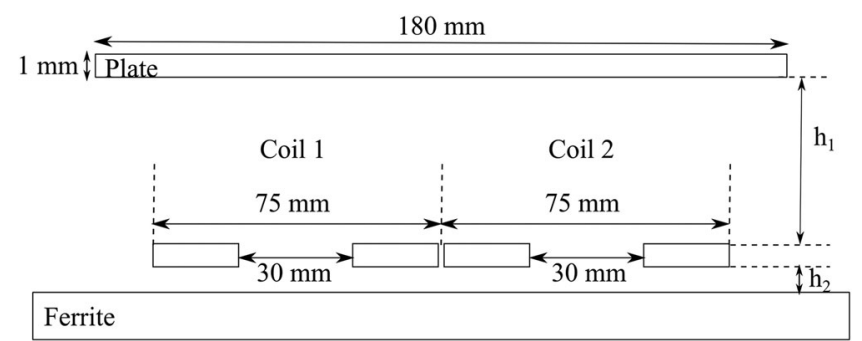

Fig. 2. Side view of our constructed heating test system.

houses, which is usually at most 20 A. Although a Litz wire with a larger number of strands can also be used in the designed coils, this leads to additional current losses and makes the coil production more costly because of wasting more wires. The eddy current losses and their changes with temperature in multiplelayer windings have been studied in the literature [14]-[18]. On the other hand, the inner circular and the outer squircle turn numbers are optimized with full electromagnetic solutions by considering available resistance and inductance values for the power supply circuit together with the coil's energy transfer efficiency.

The proposed coil architecture provides a stronger coupling when two or more of these coils are placed side by side in a square lattice. This is because of long tangential sides of the squircle geometry. Also, their rounded corners are unique for all-surface applications because they decrease destructive interaction (e.g., between the corners of diagonally located coils, which have currents in the same directions in the case of side-by-side opposite phase application). Additionally, in the proposed coil architecture, we observed that the squircle turns instead of the circular windings toward the inner rim does not further significantly improve the resulting energy transfer efficiency and mutual coupling after a certain number of the squircle turns (which was found to be 26 by systematic simulations for the induction system studied). Therefore, it does not matter to use more squircle turns toward the inner rim after this critical turn number. Circular winding is thus effectively a convenient adapter for the inner turns because production of square-shaped coils becomes increasingly difficult as their side lengths reduce, especially when thick wires are used. Also, these circular turns at the inner rim favorably reduce production cost by decreasing the total amount of copper wire used.

Fig. 2 shows the cross-sectional view of our constructed heating system. In this system, we placed two identical coils side by side and a ferrite plane covering area below the coils completely. Locating ferrites below the coils is a well-known method [13], [19]-[23], in which ferrites improve heating efficiency by directing magnetic fields below the coils toward the upper half space where the heating object is located.

In this model system, we denote the distance between the coils' top plane and the ferromagnetic stainless steel plate (AISI 430) having a diameter of $180 \mathrm{~mm}$ and a thickness of $1 \mathrm{~mm}$ to be $h_{1}=14 \mathrm{~mm}$ (due to the upper peripherals and Silargan glass) and the distance between the coils' back plane and the ferrite top plane to be $h_{2}=1 \mathrm{~mm}$ (due to the substrate layer below the 
coils used in production). Also, to prevent infrared reflections from the steel plate, which spoil thermal camera images, the top surface of the plate is covered by a nylon tape (nylon 6/6) of $0.80 \mathrm{~mm}$ in thickness.

After tuning the system's resonance frequency by using a shunt capacitor, a driving sinusoidal signal at a resonance frequency of $50 \mathrm{kHz}$ was applied by using a signal power generator. The applied current and voltage signals were measured instantaneously monitoring both their amplitudes and the phase between them by a means of oscilloscope (Agilent DSOX3012A) with a current clamp (Fluke i30) and a voltage probe. The real power supplied to the system was calculated by using (1). It is the total dissipated power in the system, which is the true power. Similarly, equivalent resistance and inductance values of the structures were calculated as

$$
\begin{aligned}
P_{\mathrm{rms}} & =\left|V_{\mathrm{rms}}\right|\left|I_{\mathrm{rms}}\right| \cos \varnothing \\
R_{\mathrm{eq}} & =\frac{\left|V_{\mathrm{rms}}\right|}{\left|I_{\mathrm{rms}}\right|} \cos \varnothing \\
L_{\mathrm{eq}} & =\frac{1}{\omega} \frac{\left|V_{\mathrm{rms}}\right|}{\left|I_{\mathrm{rms}}\right|} \sin \varnothing .
\end{aligned}
$$

Here, $P_{\mathrm{rms}}$ is the true rms power $(\mathrm{W}), R_{\mathrm{eq}}$ is the equivalent resistance $(\Omega)$, and $L_{\mathrm{eq}}$ is the equivalent inductance $(\mathrm{H}) . V_{\mathrm{rms}}$ and $I_{\mathrm{rms}}$ are the rms voltage (V) and current (A), respectively. Also, $\omega$ and $\varnothing$ are the angular frequency and phase difference between voltage and current, respectively. Total energy dissipated by the system was calculated by integrating instantaneously measured power over time. Its relation is given in

$$
E_{\text {total }}=\int P_{\mathrm{rms}} \cdot d t
$$

where $E_{\text {total }}$ is the total energy ( $\mathrm{J}$ ) dissipated by the system and $P_{\mathrm{rms}}$ is the instantaneously measured true power (W). Therefore, changes of the equivalent resistance and inductance values are investigated together with the energy dissipated by the system.

Moreover, the energy transferred to and dissipated from the stainless steel plate and the nylon tape were, respectively, calculated by using

$$
\begin{gathered}
Q_{\text {total-plate }}=Q_{\text {heating-plate }}+Q_{\text {cooling-plate }} \\
Q_{\text {total-tape }}=Q_{\text {heating-tape }}+Q_{\text {cooling-tape }}
\end{gathered}
$$

Here, $Q_{\text {total-plate }}$ and $Q_{\text {total-tape }}$ are the total dissipated energies on the plate and the tape, respectively. While heating, because of the temperature difference, there also occurs heat flow from the plate and the tape to the surrounding medium. Therefore, the total dissipated energies were calculated as a sum of the energy that increases the material's temperature and the heat that is transferred from the material to the medium (here it is air). Energies that increase the plate and tape's temperature were calculated by

$$
Q_{\text {heating }}=d \cdot V \cdot C \cdot \Delta T
$$

where $Q_{\text {heating }}$ is the heating energy $(\mathrm{J}), d$ is the material density $\left(7.74 \times 10^{-6} \mathrm{~kg} / \mathrm{mm}^{3}\right.$ for the stainless steel and $1.15 \times 10^{-6} \mathrm{~kg} / \mathrm{mm}^{3}$ for the nylon $\left.6 / 6\right), V$ is the volume of the material $(V=\pi \times 90 \mathrm{~mm} \times 90 \mathrm{~mm} \times 1 \mathrm{~mm}$ for the cylindrical steel plate and $V=\pi \times 90 \mathrm{~mm} \times 90 \mathrm{~mm} \times 0.8 \mathrm{~mm}$ for the rounded nylon tape), $C$ is the specific heat of the material (460 J $\cdot \mathrm{kg}^{-1} /{ }^{\circ} \mathrm{C}$ for the stainless steel and $1700 \mathrm{~J} \cdot \mathrm{kg}^{-1} /{ }^{\circ} \mathrm{C}$ for the nylon $6 / 6$ ), and $\Delta T$ is the temperature increase ( ${ }^{\circ} \mathrm{C}$ ). Similarly, the energy transferred from the plate and tape to the surrounding medium was calculated by

$$
Q_{\text {cooling }}=\int(h \cdot A \cdot \Delta K) d t
$$

where $Q_{\text {cooling }}$ is the transferred energy $(\mathrm{J}), h$ is the heat transfer coefficient $\left(25 \mathrm{~W} / \mathrm{m}^{2} \cdot \mathrm{K}\right.$ for the stainless steel and $0.25 \mathrm{~W} / \mathrm{m}^{2} \cdot \mathrm{K}$ for the nylon 6/6), $A$ is the surface area $(A=\pi \times 90 \mathrm{~mm} \times$ $90 \mathrm{~mm}+2 \times \pi \times 90 \mathrm{~mm} \times 1 \mathrm{~mm}$ for the steel plate and $A=$ $\pi \times 90 \mathrm{~mm} \times 90 \mathrm{~mm}+2 \times \pi \times 90 \mathrm{~mm} \times 0.8 \mathrm{~mm}$ for the nylon tape), and $\Delta K$ is the temperature difference $\left({ }^{\circ} \mathrm{C}\right)$ between the materials (plate and tape) and the surrounding medium. The transferred heat energy is the integral of the transferred power from the material to the medium over time.

Since the plate is heated up starting from regions above the coils, the temperature distribution over the tape observed at time instants is not uniform. We calculated the average temperature by simply taking integral of the temperature distribution over the tape's top surface using the thermal camera's calibrated image and divided the result by the top surface area. In our measurements, a thermal camera (FLIR A655sc) that detects infrared radiation (heat) and creates clear images based on capturing temperature difference profile is used. In (7), the temperature change $\Delta T$ is considered as the change in the calculated average temperature. Similarly, in (8) the temperature difference $\Delta K$ is taken as the difference between the calculated average temperature and the ambient temperature. In these calculations, because the steel plate's and the tape's thicknesses are so small (1.8 $\mathrm{mm}$ in total), the temperature gradient from the plate's bottom surface to the tape's top surface is ignored.

\section{RESULTS AND DISCUSSION}

For a comparative study, the same measurements were repeated with the proposed outer squircle-inner circular and the conventional circular coils. Also, to investigate its effect on the system's heating efficiency, the phase difference was applied between adjacent coils, and measurements were again repeated. Since two coils are connected in parallel, we applied $0^{\circ}$ and $180^{\circ}$ phase differences. The $0^{\circ}$ phase difference means that coils' currents flow in the same direction. The $180^{\circ}$ phase difference thus corresponds to the coils' currents flowing in the reverse directions. In practice, $0^{\circ}$ and $180^{\circ}$ phase differences can be applied simply by reversing the polarity of the terminals of the coils.

Fig. 3 shows thermal images taken at 0,30 , and $60 \mathrm{~s}$ after power is switched ON for the outer squircle-inner circular coil. Here, the plate's temperature distribution changing with time is clearly seen. In Fig. 3(a), due to a $0^{\circ}$ phase difference, coils couple destructively around their close edges and constructively around their far sides. As a result, temperature of the plate is higher in regions above the far sides of the coils than those above the close sides. On the other hand, in Fig. 3(b), due to 

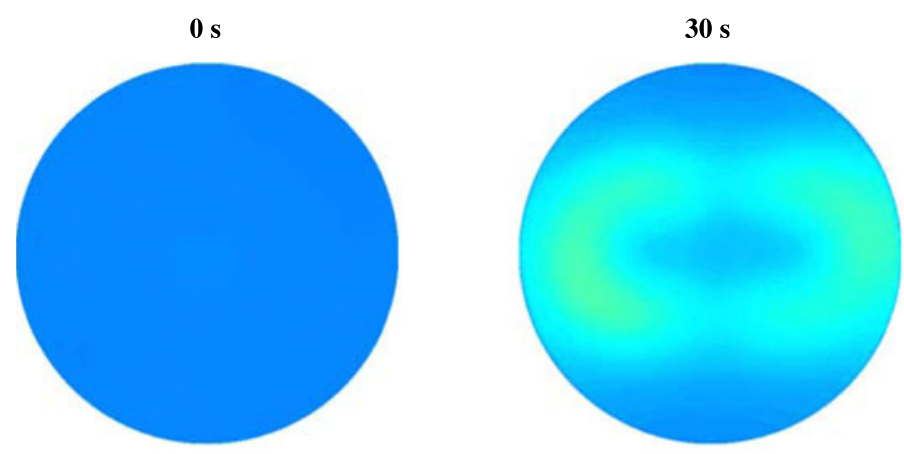

(a)

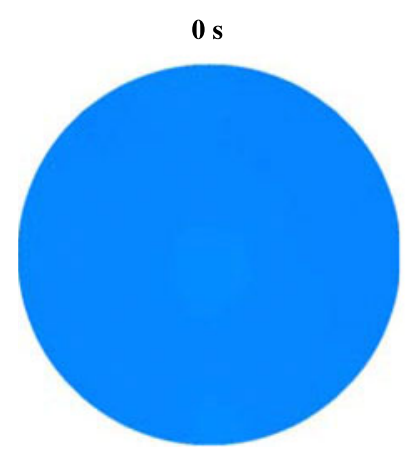

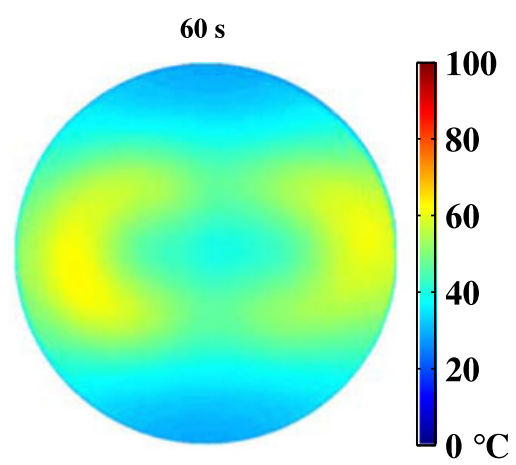

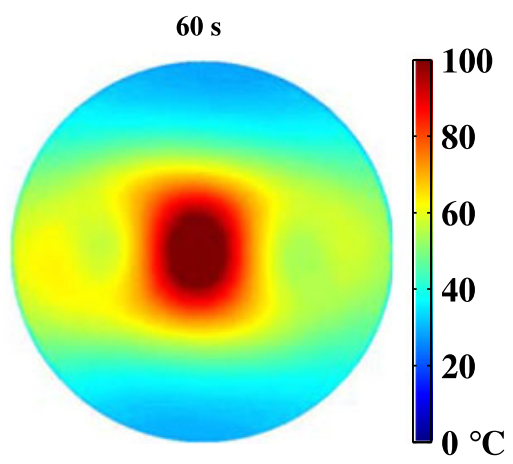

(b)

Fig. 3. Temperature distributions on the stainless steel plate at various time instants $(0,30$, and $60 \mathrm{~s})$ after power is switched oN. Here, the plate is placed above a pair of the outer squircle-inner circular coils driven with (a) a $0^{\circ}$ phase difference and (b) a $180^{\circ}$ phase difference.

(a)

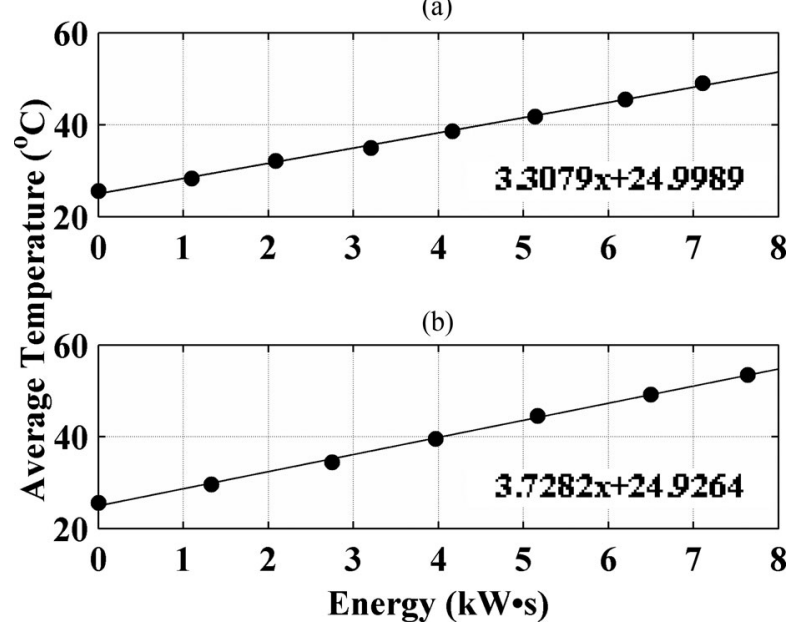

Fig. 4. Average temperature increase over the plate as a function of the energy supplied to and dissipated in the system. Here, the plate was placed above a pair of the outer squircle-inner circular coils driven with (a) a $0^{\circ}$ phase difference and (b) a $180^{\circ}$ phase difference.

a $180^{\circ}$ phase difference, fields interfere constructively at the close sides of the coils. Therefore, the plate is heated up starting from regions above the close edges of the coils.

Fig. 4 shows the average temperature as a function of the total real energy supplied to and dissipated in the system at different time instants of $10 \mathrm{~s}$ intervals, running up to a total energy level of $8 \mathrm{~kW} \cdot \mathrm{s}[0,10,20,30,40,50,60$, and $70 \mathrm{~s}$ for the $0^{\circ}$ phase difference in part (a) and $0,10,20,30,40$, 50 , and $60 \mathrm{~s}$ for the $180^{\circ}$ phase difference in part (b)]. Fitting curves indicate that the temperature increases linearly at a rate of approximately $3.31{ }^{\circ} \mathrm{C} /(\mathrm{kW} \cdot \mathrm{s})$ for the $0^{\circ}$ phase difference and $3.73{ }^{\circ} \mathrm{C} /(\mathrm{kW} \cdot \mathrm{s})$ for the $180^{\circ}$ phase difference. In other words, with $1000 \mathrm{~W} \cdot \mathrm{s}$ input energy dissipated in the system, the plate's temperature increases by $3.31{ }^{\circ} \mathrm{C}$ and $3.73{ }^{\circ} \mathrm{C}$ on average when the proposed outer squircle-inner circular coils are driven with the $0^{\circ}$ and $180^{\circ}$ phase differences. This is a useful figure of merit for efficiency comparisons.

Another figure of merit that we used in our study for efficiency is the ratio of the energy transferred to and dissipated on the plate/tape $\left(Q_{\text {total-plate }}+Q_{\text {total-tape }}\right)$ to the total energy dissipated in the system $\left(E_{\text {total }}\right)$. In the system, when a $0^{\circ}$ phase difference exists between the coils, then by using (7), we found the heating energies dissipated on the stainless steel plate and the nylon tape over $70 \mathrm{~s}$ to be equal to 2130.86 and $936.04 \mathrm{~W} \cdot \mathrm{s}$, respectively. Similarly, by using (8), energies transferred from the plate and the tape to the air medium were calculated to be 547.45 and $5.47 \mathrm{~W} \cdot \mathrm{s}$, respectively. In addition, the total real energy supplied to the system over $70 \mathrm{~s}$ was $7109.93 \mathrm{~W} \cdot \mathrm{s}$. Therefore, in total $0.509[(2130.86+936.04+547.45+5.47) / 7109.93]$ ratio of the real energy supplied to the system was transferred to and dissipated on the plate/tape. Similarly, in the system, when the coils 
(a)

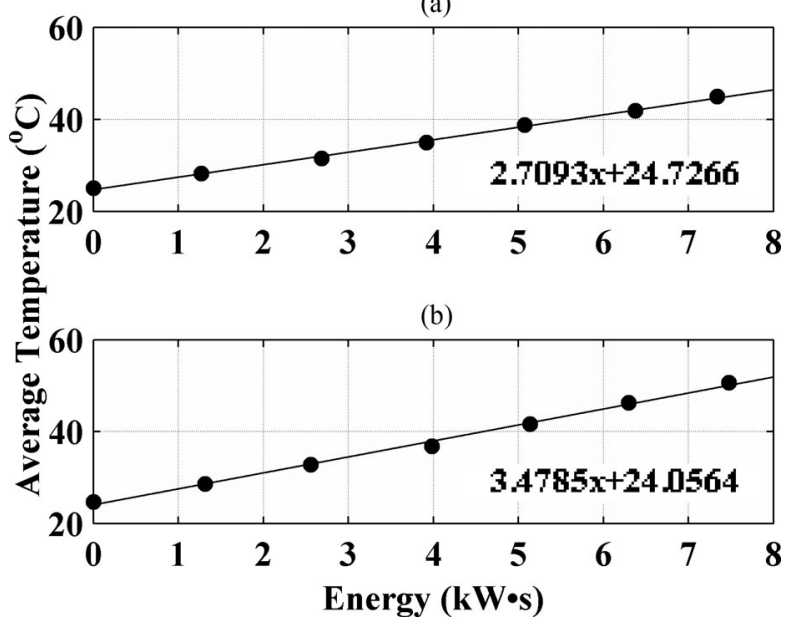

Fig. 5. Average temperature increase over the plate as a function of the energy supplied to and dissipated in the system. Here, the plate was placed above a pair of the circular coils driven with (a) a $0^{\circ}$ phase difference and (b) a $180^{\circ}$ phase difference.

TABLE I

COMPARISON OF PROPOSED OUTER SQUIRCLE-INNER CIRCULAR AND CONVENTIONAL CIRCULAR COILS

\begin{tabular}{lccc}
\hline \hline Coils & Phase & $\begin{array}{c}\text { Temperature increase } \\
\text { rate }\left[{ }^{\circ} \mathrm{C} /(\mathrm{kW} \cdot \mathrm{s})\right]\end{array}$ & $\begin{array}{c}\text { Energy transfer } \\
\text { ratio }\end{array}$ \\
\hline Outer squircle-inner circular & $0^{\circ}$ & 3.31 & 0.509 \\
& $180^{\circ}$ & 3.73 & 0.562 \\
Circular & $0^{\circ}$ & 2.71 & 0.409 \\
& $180^{\circ}$ & 3.48 & 0.524 \\
\hline \hline
\end{tabular}

were driven with a $180^{\circ}$ phase difference, the total energy dissipated on the plate/tape and the real energy supplied to the system over $60 \mathrm{~s}$ were found to be 4289.15 and $7637.50 \mathrm{~W} \cdot \mathrm{s}$, respectively. Resultantly, $0.562(4289.15$ / 7637.50) ratio of the real energy supplied to the system was transferred to the plate/tape.

We replaced the outer squircle-inner circular coils with the conventional circular coils and measurements were repeated with the $0^{\circ}$ and $180^{\circ}$ phase differences between coils. Again, in Fig. 5 dots represent the data points of the calculated average temperatures and the total real energies supplied to and dissipated in the system at time instants $(0,10,20,30,40$, 50 , and $60 \mathrm{~s}$ ) of which thermal images were captured. Also, in the figure the fitting curves give slopes of approximately $2.71{ }^{\circ} \mathrm{C} /(\mathrm{kW} \cdot \mathrm{s})$ and $3.48{ }^{\circ} \mathrm{C} /(\mathrm{kW} \cdot \mathrm{s})$ for the $0^{\circ}$ and $180^{\circ}$ phase differences, respectively. These results (shown in Fig. 5) are tabulated in Table I together with those of outer squircle-inner circular coils (given in Fig. 4).

As shown in Table I, the results are consistently better for the outer squircle-inner circular coils than for the circular coils. This means that the proposed coils lead to higher heating efficiency than the conventional circular coils. In addition, applying the $180^{\circ}$ phase difference increases the efficiency. The outer squircle-inner circular coil reaches the highest heating rate of all when driven with the $180^{\circ}$ phase difference. The energy transfer ratio found for the outer squircle-inner circular coil with the $180^{\circ}$ phase difference is $37.4 \%$ higher than the ratio calculated for the circular coil with the $0^{\circ}$ phase difference.

The high efficiencies in Table I obtained with the outer squircle-inner circular coil also imply that the proposed outer squircle-inner circular coils are less sensitive to phase differences than conventional circular coils. This might be due to unusual geometry of the outer squircle-inner circular coils. Long tangential sides in squircle geometry increase interaction between adjacent coils.

In Fig. 6, temperature distributions on the plate with equal amount of real energies supplied to the systems are illustrated. As in previous figures, in Fig. 6 temperature of the plate was higher above the far sides of the coils than above the close sides when $0^{\circ}$ phase difference exists between coil currents. On the other hand, temperature of the plate was the highest at its center when the coils were driven with opposite phases. In all of these thermal camera snapshots, the temperature of the plate was higher for the newly designed outer squircle-inner circular coils than the conventional circular coils. In addition, for comparison purposes, the temperature increase of the plate for all systems is presented in Fig. 7 across a fixed line that passes through the center of the plate. This line can be visualized as a horizontal line that passes through the middle of the plate and the coils' heating areas in Fig. 6. Also, temperature increase is the difference between the plate temperature after $5 \mathrm{~kW} \cdot \mathrm{s}$ total real energy was supplied to the system and its initial temperature when no energy was supplied. In Fig. 7, legends S0 and S180 represent systems in which the outer squircle-inner circular coil pair was driven with $0^{\circ}$ and $180^{\circ}$ phase differences, respectively. Similarly, legends $\mathrm{C} 0$ and $\mathrm{C} 180$ denote the systems in which the circular coil pair was driven with $0^{\circ}$ and $180^{\circ}$ phase differences, respectively. To visually aid the reader, the temperature profiles were plotted to have their central point at the position $0 \mathrm{~mm}$.

In Fig. 7, pairwise comparisons of profile S0 with profile $\mathrm{C} 0$ and $\mathrm{S} 180$ with $\mathrm{C} 180$ indicate that the temperature increase in the plate is higher in systems where newly designed outer squircle-inner circular coils were used instead of the conventional circular coils. Since the total real energy supplied to the system is constant, similar trends were observed for the energy transfer ratio. These results agree well with our previous results given in Table I.

Furthermore, to investigate the availability of the proposed outer squircle-inner circular coil for the power supply circuitry used to drive conventional coils, equivalent resistance and inductance values of the designs were calculated using (2) and (3), respectively. For this, voltage and current signals applied to the system were measured instantaneously over time. In Fig. 8, measured electrical parameters including applied voltage, rated current, and true power levels are presented as a function of the supplied real energy. In these and following figures, data points are denoted with markers and curves show the first-order [in Fig. 8(a)] and the second-order [in Fig. 8(b) and (c)] fittings. As in Fig. 7, here S0 and S180 again represent the systems where the outer squircle-inner circular coil pair is driven with the $0^{\circ}$ and $180^{\circ}$ phase differences, respectively. Similarly, C0 and C180 correspond to the systems where the circular coil pair is driven with the $0^{\circ}$ and $180^{\circ}$ phase differences, respectively. 


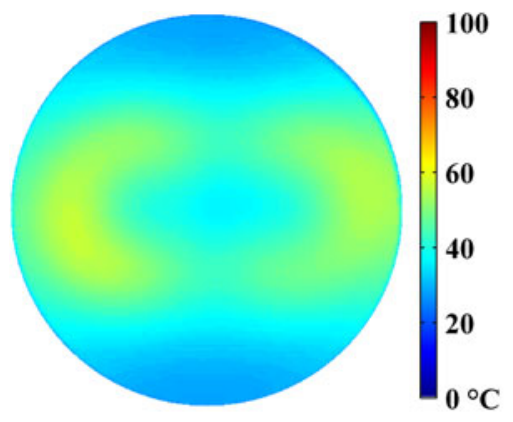

(a)

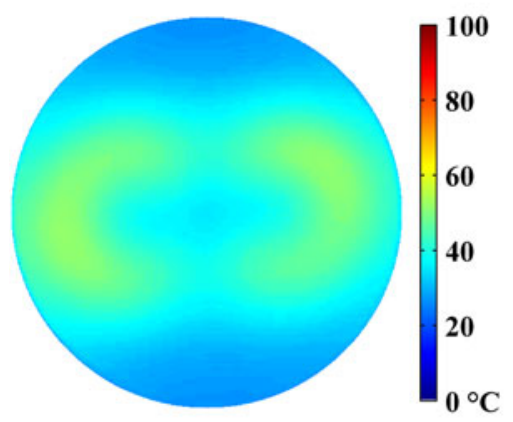

(c)

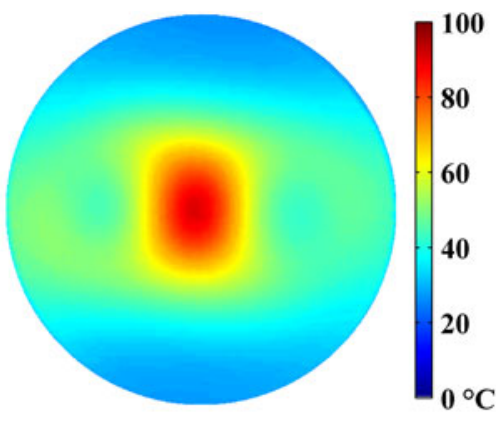

(b)

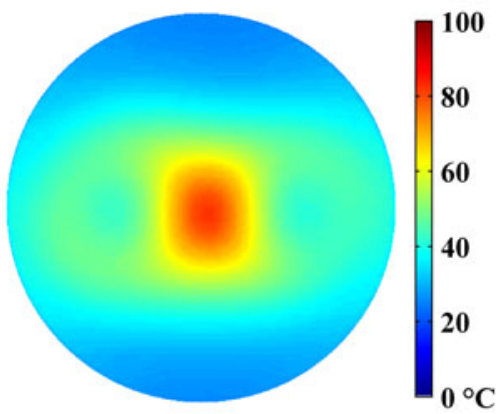

(d)

Fig. 6. Temperature distributions on the stainless steel plate with a total supplied real energy of approximately $5 \mathrm{~kW} \cdot \mathrm{s}$ : for the outer squircle-inner circular coil pair driven with (a) a $0^{\circ}$ phase difference and (b) a $180^{\circ}$ phase difference and the conventional circular coil pair driven with (c) a $0^{\circ}$ phase difference and (d) a $180^{\circ}$ phase difference.

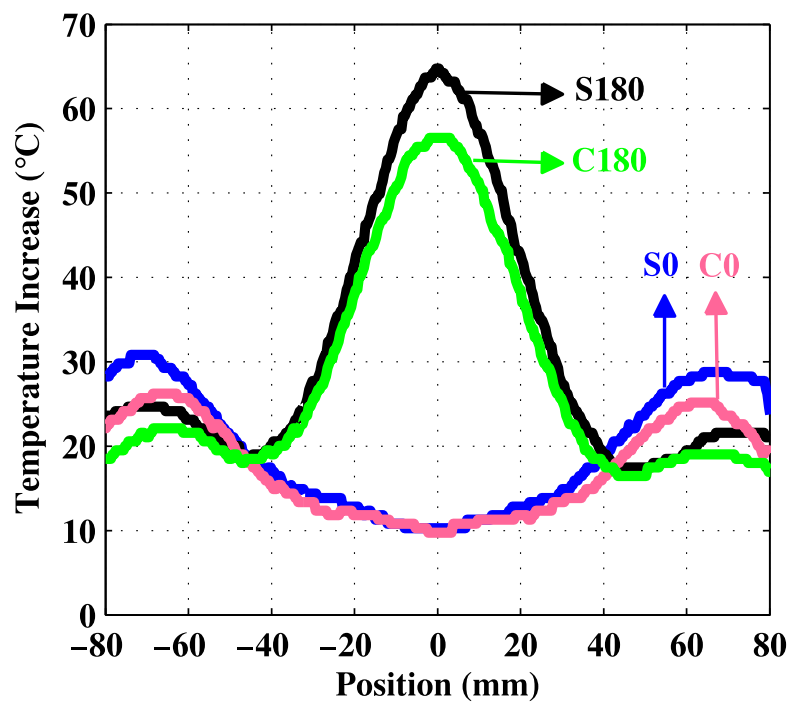

Fig. 7. Temperature increase on constant line that passes through the middle of the plate. Here, approximately $5 \mathrm{~kW} \cdot \mathrm{s}$ total real energies are supplied to the systems.

In our system, supply circuit behaves as a voltage source [see Fig. 8(a)]. For fair comparison, input currents are set to similar values but with energy the rated current values increase. This is explained with the decrease in system impedance. In addition, the power supplied to and dissipated in the system decreases with energy, too. In Fig. 8, despite their similar rated current values, the dissipated power is noticeably lower in the system in which the outer squircle-inner circular coil pair is driven with the $0^{\circ}$ phase difference, than those in the other systems. These changes are related to variations in the resistance and the inductance. Resistance and inductance values of the system are important not only for the power transmission but also the vessel detection. Results are shown in Figs. 9 and 10.

In Fig. 9, equivalent resistances of the system have close values. In particular, the system using newly designed outer squircle-inner circular coil pair driven with the $180^{\circ}$ phase difference has very similar equivalent resistance values to those systems in which circular coils are used. This is valuable because the new coil design having resistance values close to those of the conventional coil systems is desired for power circuitry.

The low resistance values in the system in which the outer squircle-inner circular coils have $0^{\circ}$ phase difference lead to a decrease in the supply power [see Fig. 8(c)]. Resultantly, in Fig. 4, a slower enhancement in average temperature over time was observed in the $0^{\circ}$ phase difference measurement. For example, in the system in which the outer squircle-inner circular coils had $0^{\circ}$ phase difference, the average temperature reached $50{ }^{\circ} \mathrm{C}$ around $75 \mathrm{~s}$ but in the system in which the designed coils were driven with the $180^{\circ}$ phase difference the average temperature reached $50{ }^{\circ} \mathrm{C}$ in $52 \mathrm{~s}$. Slow heating of the plate causes increased heat loss from the plate to the medium $\left[Q_{\text {cooling }}\right.$ in (8) $]$. The low value of the temperature increase rate in Table I for the outer squircle-inner circular coils having a $0^{\circ}$ phase difference with respect to the energy transfer ratio is because of this high amount of heat loss to the medium. 


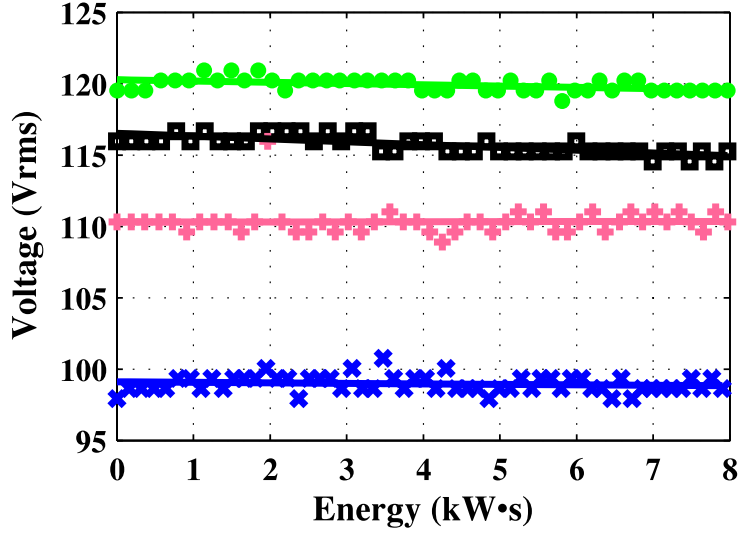

(a)

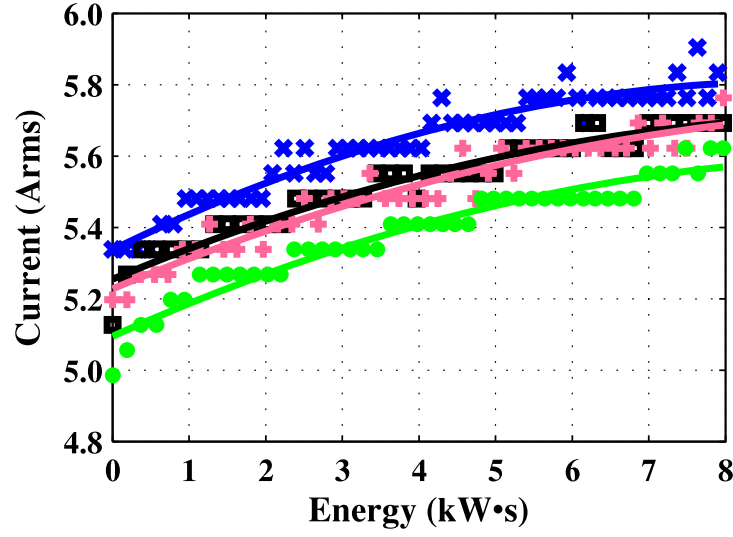

(b)

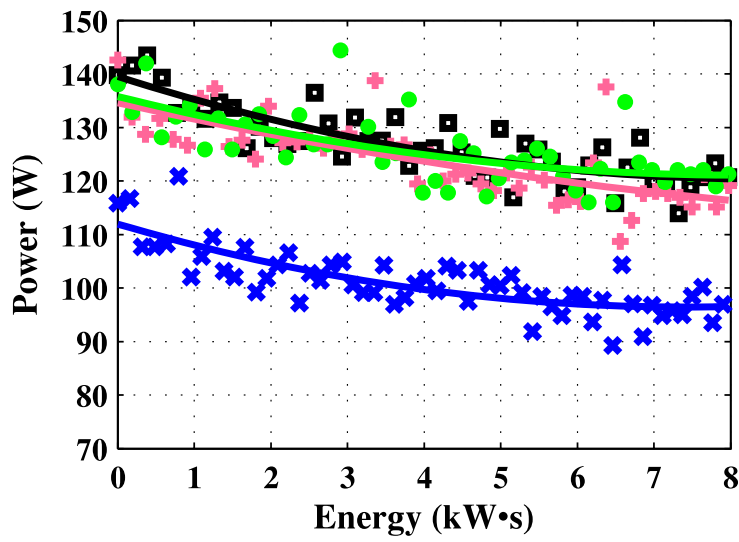

\begin{tabular}{|ll|}
\hline$*$ & S0 \\
$\square$ & S180 \\
+ & C0 \\
$*$ & C180
\end{tabular}

(c)

Fig. 8. Measured electrical parameters: (a) applied voltage, (b) rated current, and (c) true power levels as a function of the real energy supplied to the system.

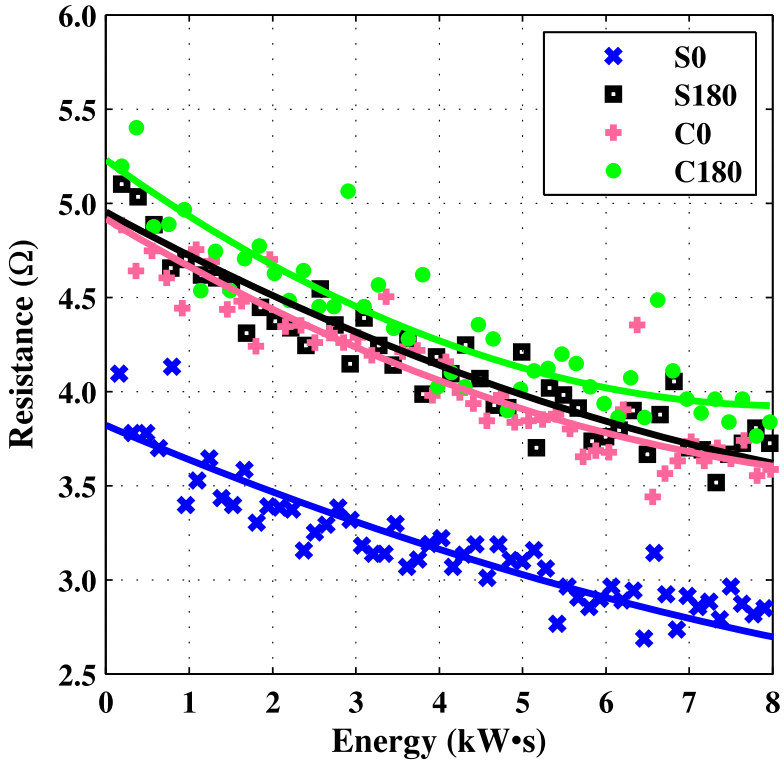

Fig. 9. Systems equivalent resistance as a function of the supplied real energy.

Moreover, in Fig. 10, equivalent inductance values are lower in the systems represented with S0 and S180 than those of $\mathrm{C} 0$ and $\mathrm{C} 180$, respectively. However, similar to the resistance

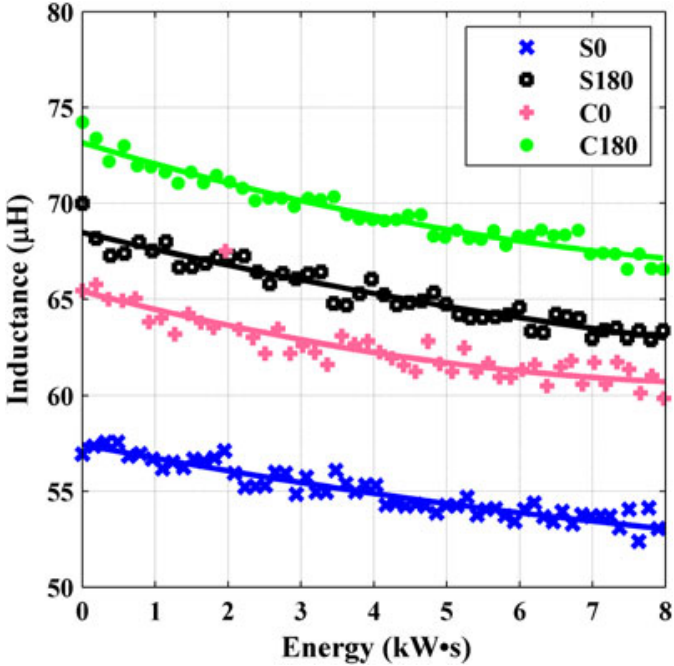

Fig. 10. Systems equivalent inductance as a function of the supplied real energy.

trends, inductance values of the systems are still close to each other. Again, this is good for the power circuitry and vessel detection.

In the figures, equivalent resistance and inductance of the systems decrease with energy. Although electrical resistivity and 


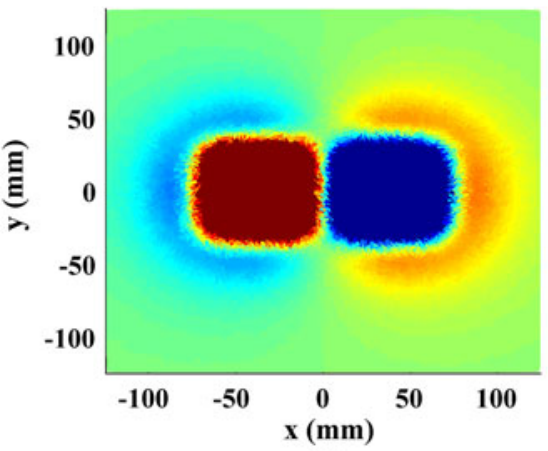

(a)

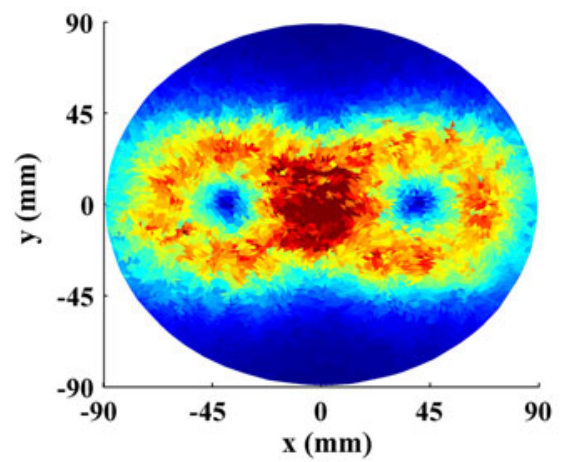

(c)

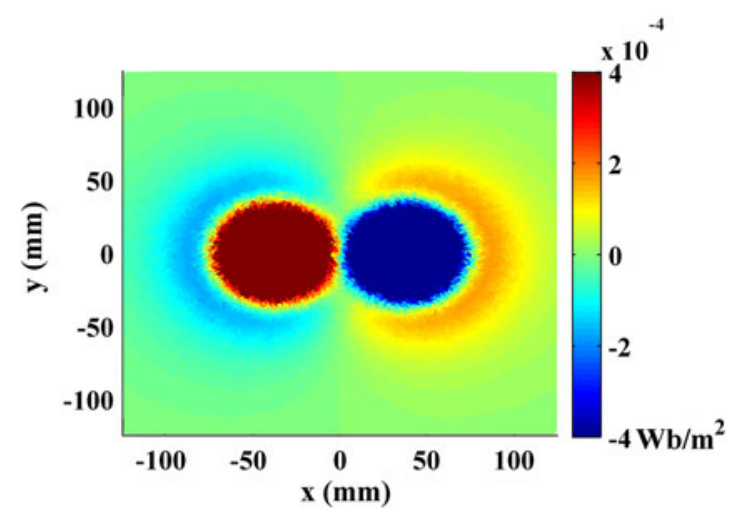

(b)

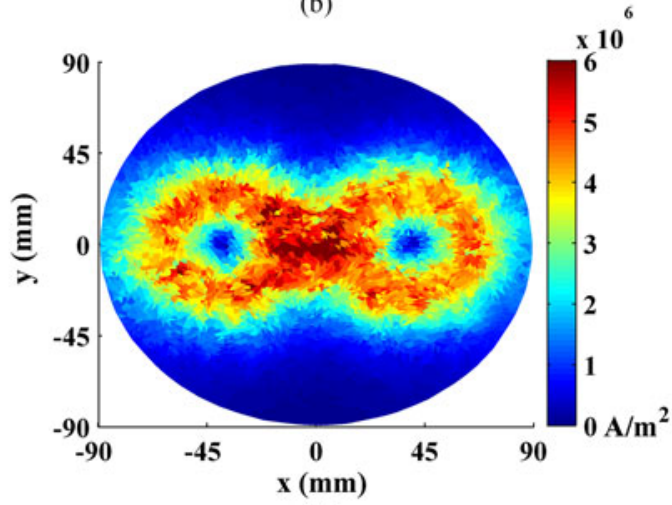

(d)

Fig. 11. Magnetic flux distributions normal to the plate's bottom surface $14 \mathrm{~mm}$ above from pairs of (a) outer squircle-inner circular and (b) circular coils and tangential current intensity distributions on the plate's bottom surface with pairs of (c) outer squircle-inner circular and (d) circular coils. Here, $180^{\circ}$ phase difference exists between the coils. Note that the axis scaling in the subfigures is not always the same.

magnetic permeability of the stainless steel and iron compounds increase with temperature (down to the Curie point) [24], [25], this reduction can be explained with decreased coupling. Flux generated by the coils decreases as their temperature increases. This results in reduction in the system's equivalent inductance. Similarly, because coupling decreases, the total power dissipation in the system reduces, too. This might be the reason of the observed decrease in the system's equivalent resistance.

The system was further analyzed with three-dimensional (3-D) numerical simulations. The magnetic flux produced by the coils and the resulting induced eddy currents on the plate were calculated. In the simulations, pairs of the proposed outer squircle-inner circular and the circular coils were placed side by side and a steel plate having cylindrical shape with $1 \mathrm{~mm}$ thickness and $180 \mathrm{~mm}$ diameter was located $14 \mathrm{~mm}$ above from the coil. To decrease the memory requirement, the ferrite plane located below the coils in the measurements was not placed in the simulated system. The rated current values initially supplied to the coils in measurements were around 5 A [see Fig. 8(b)]. Since coils were connected in parallel, currents flowing on each of the coil were approximately $2.5 \mathrm{~A}$. Therefore, in the simulations each coil was driven by separate current sources alternating at $50 \mathrm{KHz}$ frequency with $2.5 \mathrm{~A}$ amplitude and $0^{\circ}$ and $180^{\circ}$ phase differences. The magnetic flux distribution normal to the plate's bottom surface (14 mm above from the coils) and amplitude distribution of the induced eddy currents tangential on the plate's bottom surface are shown in Fig. 11.

In the figure, because the coils are driven with $180^{\circ}$ phase differences, the magnetic fluxes are in reverse directions [see Fig. 11(a) and (b)]. Therefore, the induced eddy currents are in reverse directions, too. For instance, current induced on one side is in clockwise direction and on the other side it is in counterclockwise direction. At the center, these induced eddy currents are in the same direction, both in the $+y$ direction in the figures. Therefore, the induced eddy currents couple constructively at the plate's center. This is clearly seen in current density distributions in Fig. 11(c) and (d). In addition, the magnetic fluxes have distributions with shapes similar to those of the coils, i.e., squircle shape in (a) and circular shape in (b). The reverse directed magnetic fluxes around these shapes are because of magnetic fields' curling. However, these curled magnetic fluxes have smaller magnitudes than fluxes that directly hit the plate's bottom surface. Therefore, the induced eddy currents on the plate's bottom surface are mostly induced by the magnetic fluxes directly impingent on the plate. In systems where the proposed outer squircle-inner circular coils are used, the magnetic fluxes and the induced eddy currents have longer tangential sides that increase the interaction than those in systems in which the circular coils are used. Long tangential sides of the magnetic fluxes that increase the interaction and the high current densities at the plate's center in the system in which the 
proposed outer squircle-inner circular coils are used are clearly seen in the figure.

In the simulations, contributions of the plate to the system's equivalent resistance were calculated, too. This is important because for a constant current there is a linear relation between the plate's contribution to the resistance value and the power transferred to and dissipated in the plate. Increase in the system's equivalent resistance with the plate's placement, where a pair of the outer squircle-inner circular coils are driven by currents having a $180^{\circ}$ phase difference, was calculated as $4.721 \Omega$. On the other hand, in the system in which the circular coils are driven by currents having a $0^{\circ}$ phase difference, increase in the system's resistance with the plate's placement was found to be $3.499 \Omega$. Therefore, the transferred power to the plate calculated in the system in which the proposed outer squircle-inner circular coils have a $180^{\circ}$ phase difference is $34.9 \%$ higher than that found in the system in which the circular coils have a $0^{\circ}$ phase difference. This enhancement is close to the value that we experimentally accomplished in the measurements $(37.4 \%)$.

System simulations were obtained with plates made of different materials, having various sizes and being located at different distances from the coils. These loading parameters were selected to cover the entire range specific to induction heating hob systems. In all these simulations, the highest efficiency was found in the systems where the proposed outer squircle-inner circular coils were used and driven with a $180^{\circ}$ phase difference. The proposed outer squircle-inner circular coils were thus found to exhibit better performance than that of conventional circular coils for all possible loading conditions common to induction heating application.

\section{CONCLUSION}

In this paper, a newly proposed architecture of outer squircleinner circular coils was presented. Energy transfer and heating efficiencies were measured and results were compared with those of conventional circular coils. Also, the effect of phase difference application between the coils on heating efficiency was investigated with comparative $0^{\circ}$ and $180^{\circ}$ phase difference measurements. In experiments, the proposed outer squircleinner circular coils exhibited higher heating efficiencies than those obtained with the conventional circular coils, with an overall enhancement of $37.4 \%$ in the system efficiency. In addition, driving coils with a $180^{\circ}$ phase difference was shown to further increase the efficiency. Therefore, the highest efficiency was obtained in the case in which the outer squircle-inner circular coil pair is driven with a $180^{\circ}$ phase difference, reaching a maximum level of. The equivalent inductance and resistance of the systems were measured, too. Systems with the proposed outer squircleinner circular coils sustain similar inductance and resistance values to those of the conventional circular coil systems, which is favorable for power circuitry and vessel detection. Measurement results were also verified with 3-D numerical simulations. The finding of this study are beneficial for induction cooking ovens in terms of efficient heating of different-sized vessels and full coverage of the oven surface. Also, another important ad- vantage is relative adaptability to phase difference applications. Current extension of this work focuses on the placement of coils in an array for all-surface heating.

\section{REFERENCES}

[1] J. Acero et al., "Domestic induction appliances: An overview of recent research," IEEE Ind. Appl. Mag., vol. 16, no. 2, pp. 39-47, Mar./Apr. 2010, doi: 10.1109/MIAS.2009.935495.

[2] S. Wang, K. Izaki, I. Hirota, H. Yamashita, H. Omori, and M. Nakaoka, "Induction-heated cooking appliance using new quasi-resonant ZVSPWM inverter with power factor correction," IEEE Trans. Ind. Appl., vol. 34, no. 4, pp. 705-712, Jul./Aug. 1998, doi: 10.1109/28.703961.

[3] J. M. Burdio, F. Monterde, J. R. Garcia, L. A. Barragan, and A. Martinez, "A two-output series-resonant inverter for induction-heating cooking appliances," IEEE Trans. Power Electron., vol. 20, no. 4, pp. 815-822, Jul. 2005, doi: 10.1109/TPEL.2005.850925.

[4] O. Lucia, J. M. Burdio, I. Millan, J. Acero, and L. A. Barragan, "Efficiency oriented design of ZVS half-bridge series resonant inverter with variable frequency duty cycle control," IEEE Trans. Power Electron., vol. 25, no. 7, pp. 1671-1674, Jul. 2010, doi: 10.1109/TPEL.2010.2042461.

[5] J. Acero, C. Carretero, I. Millan, O. Lucia, R. Alonso, and J. M. Burdio, "Analysis and modeling of planar concentric windings forming adaptable-diameter burners for induction heating appliances," IEEE Trans. Power Electron., vol. 26, no. 5, pp. 1546-1558, May 2011, doi: 10.1109/TPEL.2010.2085453.

[6] C. Carretero, O. Lucia, J. Acero, and J. M. Burdio, "Phase-shift control of dual half-bridge inverter feeding coupled loads for induction heating purposes," IET Electron. Lett., vol. 47, no. 11, pp. 670-671, May 2011, doi: 10.1049/el.2011.1114

[7] I. Millan, J. M. Burdio, J. Acero, O. Lucia, and D. Palacios, "Resonant inverter topologies for three concentric planar windings applied to domestic induction heating," IET Electron. Lett., vol. 46, no. 17, pp. 1225-1226, Aug. 2010, doi: 10.1049/el.2010.1197.

[8] F. Forest, S. Faucher, J. Y. Gaspard, D. Montloup, J. J. Huselstein, and C. Joubert, "Frequency-synchronized resonant converters for the supply of multiwinding coils in induction cooking appliances," IEEE Trans. Ind. Electron., vol. 54, no. 1, pp. 441-452, Feb. 2007, doi: 10.1109/TIE.2006.888797

[9] J. Acero, C. Carretero, O. Lucia, R. Alonso, and J. M. Burdio, "Mutual impedance of small ring-type coils for multiwinding induction heating appliances," IEEE Trans. Power Electron., vol. 28, no. 2, pp. 1025-1035, Feb. 2013, doi: 10.1109/TPEL.2012.2205270.

[10] D. Fournier, E. Merliot, and A. Roux, "Assembling module of induction coils of a induction heating cooking area and cooking area including the said modules," Eur. Patent EP 1575336 B1, Jan. 27, 2010.

[11] K. Leidig and M. Herzog, "Induction cooking hob," Eur. Patent EP 2265088 B1, Jun. 27, 2012.

[12] L. C. Meng, K. W. E. Cheng, and W. M. Wang, "Thermal impacts of electromagnetic proximity effects in induction cooking system with distributed planar multicoils," IEEE Trans. Magn., vol. 47, no. 10, pp. 3212-3215, Oct. 2011, doi: 10.1109/TMAG.2011.2148103.

[13] A. Roux, "Induction device comprising multiple individual coils for induction heating plates," U.S. Patent US 2009/0020526 A1, Jan. 22, 2009.

[14] R. P. Wojda and M. K. Kazimierczuk, "Winding resistance of litz-wire and multi-strand inductors," IET Power Electron., vol. 5, no. 2, pp. 257-268, Feb. 2012, doi 10.1049/iet-pel.2010.0359.

[15] R. P. Wojda and M. K. Kazimierczuk, "Analytical winding size optimisation for different conductor shapes using Ampere's Law," IET Power Electron., vol. 6, no. 6, pp. 1058-1068, Jul. 2013, doi: 10.1049/ietpel.2011.0415

[16] R. P. Wojda and M. K. Kazimierczuk, "Analytical optimization of solid-round-wire windings," IEEE Trans. Ind. Electron., vol. 60, no. 3 , pp. 1033-1041, Mar. 2013, doi: 10.1109/TIE.2012.2189543.

[17] A. Ayachit and M. K. Kazimierczuk, "Thermal effects on inductor winding resistance at high frequencies," IEEE Magn. Lett., vol. 4, Nov. 2013 , Art no. 0500304, doi: 10.1109/LMAG.2013.2286582

[18] M. K. Kazimierczuk, High-Frequency Magnetic Components, 2nd ed. Chichester, U.K.: Wiley, 2014

[19] J. Acero, P. J. Hernandez, J. M. Burdio, R. Alonso, and L. A. Barragan, "Simple resistance calculation in litz-wire planar windings for induction cooking appliances," IEEE Trans. Magn., vol. 41, no. 4, pp. 1280-1288, Apr. 2005, doi: 10.1109/TMAG.2005.844844. 
[20] W. A. Roshen and D. E. Turcotte, "Planar inductors on magnetic substrates," IEEE Trans. Magn., vol. 24, no. 6, pp. 3213-3216, Nov. 1988, doi 10.1109/20.92379.

[21] W. A. Roshen, "Effect of finite thickness of magnetic substrate on planar inductors," IEEE Trans. Magn., vol. 26, no. 1, pp. 270-275, Jan. 1990, doi: $10.1109 / 20.50553$

[22] J. Acero, R. Alonso, J. M. Burdio, and L. A. Barragan, "Enhancement of induction heating performance by sandwiched planar windings," IET Electron. Lett., vol. 42, no. 4, pp. 241-242, Feb. 2006, doi: 10.1049/el:20064123

[23] I. Hirota et al., "Induction heating device," U.S. Patent 7057144 B2, Jun. 6, 2006.

[24] J. R. Davis, "Properties of stainless steels," in ASM Speciality Handbook: Stainless Steels. Materials Park, OH, USA: ASM International, 1994, pp. 489-495.

[25] M. Enokizono, "Numerical analysis of high-frequency induction heating including temperature dependence of material characteristics," IEEE Trans. Magn., vol. 31, no. 4, pp. 2438-2444, Jul. 1995, doi: $10.1109 / 20.390154$

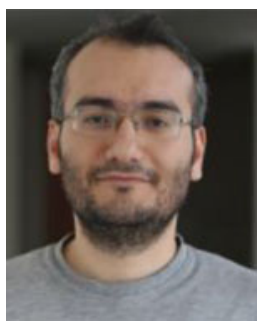

Veli Tayfun Kilic (S'14) was born in Adana, Turkey, on August 1, 1987. He received the B.S. and M.S. degrees in electrical and electronics engineering from Bilkent University, Ankara, Turkey, in 2009 and 2011, respectively, where he is currently working toward the Ph.D. degree under the supervision of Prof. H. V. Demir. His research focus is high-efficiency inductive energy transfer and applications.

Since 2009, he has been a Research Assistant with the Electrical and Electronics Engineering Department, Bilkent University. From 2012 to 2013, he was a Design Engineer with the Mikes Company. Since 2014, he has been a Researcher with TUBITAK, the Scientific and Technological Research Council of Turkey, Ankara. His research interests include radiofrequency systems, near-field and far-field electromagnetic coupling, radio-frequency antennas, plasmonic antennas, and radio-frequency circuits.

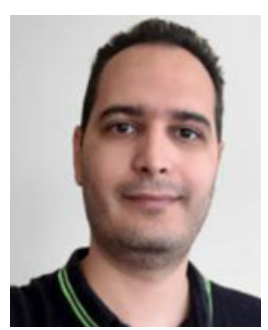

Emre Unal received the B.S. degree in electrical and electronics engineering from Hacettepe University, Ankara, Turkey, in 2005.

$\mathrm{He}$ is a full-time Research Engineer under the supervision of Prof. H. V. Demir with the Institute of Materials Science and Nanotechnology, Bilkent University, Ankara, where he is working on the development of microwave and optoelectronic devices.

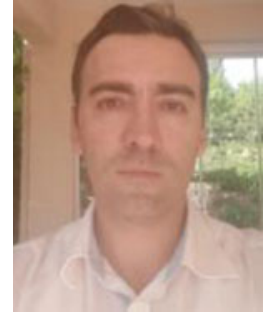

Erdal Gonendik received the B.S. degree from the Middle East Technical University, Ankara, Turkey, and the M.S. degree from Bilkent University, Ankara, both in electrical and electronics engineering department, in 2001 and 2014, respectively. $\mathrm{He}$ is currently working toward the Ph.D. degree at Bilkent University in the Department of Electrical and Electronics Engineering.

$\mathrm{He}$ is also a Specialist Engineer with ASELSAN, a leading electronics and electronic systems company in Turkey.

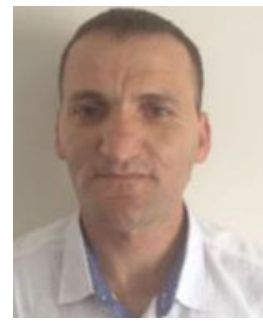

Namik Yilmaz received the B.S. and M.S. degrees in electrical engineering from Istanbul Technical University, Istanbul, Turkey, in 1997 and 2000 , respectively, where he is currently working toward the Ph.D. degree in electrical engineering.

$\mathrm{He}$ has been with the Power Electronics Group of Arcelik A.S., Istanbul, Turkey, since 2000. His main interests include design of motor control hardware and algorithms, design of resonant inverter topologies, coils, and algorithms for domestic induction cooking and microwave heating.

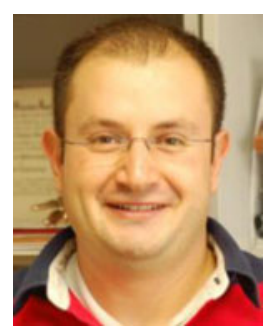

Hilmi Volkan Demir (M'04-SM'11) received the B.S. degree in electrical and electronics engineering from Bilkent University, Ankara, Turkey, in 1998, and the M.S. and Ph.D. degrees in electrical engineering from Stanford University, Stanford, CA, USA, in 2000 and 2004, respectively.

In September 2004, he joined Bilkent University, where he is currently a Professor with joint appointments in the Department of Electrical and Electronics Engineering and the Department of Physics and is also with the Institute of Materials Science and Nanotechnology. Concurrently, he is a Fellow of the National Research Foundation in Singapore and a Professor at Nanyang Technological University, Singapore. His research interests include the development of innovative optoelectronic and RF devices.

Prof. Demir received the European Union Marie Curie Fellowship, the Turkish National Academy of Sciences Distinguished Young Scientist Award, the European Science Foundation-European Young Investigator Award, and the Nanyang Award for Research Excellence. 